\title{
COMMENT
}

\section{Commentary on "The point-of-care Bilistick method has very short turn-around-time and high accuracy at lower cut-off levels to predict laboratory-measured TSB."}

\author{
Carlo Dani ${ }^{1,2}$ \\ Pediatric Research (2019) 86:147-148; https://doi.org/10.1038/s41390-019-0378-8
}

Hyperbilirubinemia is the most frequent clinical problem neonatologists must deal with during the newborn period because it can affect more than $60 \%$ of late preterm and term infants. Hyperbilirubinemia represents a relevant workload for neonatal units and it has been observed to be the primary reason for about $5 \%$ of hospital admissions and $22 \%$ of re-admissions. ${ }^{1}$ High levels of total serum bilirubin (TSB) can be toxic to the central nervous system. Gale et al., using data from the National Neonatal Research Database, have reported that bilirubin encephalopathy was the sixth cause of neonatal brain injuries in the United Kingdom during the period of 2012-2015 with a rate of about one case per 100000 live births per year. ${ }^{2}$ Recently, the systematic nomenclature of bilirubin-induced brain injuries has been updated: the term acute bilirubin encephalopathy should be used only to designate the acute phase of bilirubin encephalopathy, while the term kernicterus spectrum disorders (KSDs) should be used to encompass all the neurological sequelae of bilirubin neurotoxicity. ${ }^{3}$ Moreover, KSDs should be classified as auditorypredominant, motor-predominant, or both auditory and motor dysfunction subtypes, and on the basis of clinical disorder severity (mild, moderate, and severe)..$^{3}$

Several national recommendations have been developed to prevent KSDs by establishing TSB threshold levels associated with risk for neurological damage at which to start phototherapy treatment, the first-line treatment for neonatal hyperbilirubinemia and, eventually, to perform exchange transfusions. Thus, the question of TSB monitoring is very important and having accurate methods for its assessment is crucial. In the clinical practice, TSB is generally measured on plasma using a diazotization reaction or on whole blood using a blood gas analyzer with a spectrophotometer module. ${ }^{4}$ Moreover, to limit frequent and painful blood sampling, transcutaneous bilirubin (TCB) measurement, which is a less invasive method, has widespread use. This measurement is performed using spectral reflectance instruments. However, the use of TcB measurements has some limitations such as $\pm 15-20 \%$ imprecision depending on gestational age, skin pigmentation, TSB $>188 \mu \mathrm{mol} / \mathrm{L}(10.9 \mathrm{mg} / \mathrm{dL})$, and its inaccuracy during phototherapy. ${ }^{4}$

In a recent study Boo et al. report a new device, namely the Bilistick System (Bilimetrix s.r.l., Trieste, Italy), that was used in a large population of jaundiced term infants in Malaysia to compare its accuracy with the gold standard laboratory TSB measurement. ${ }^{5}$ The system is a point-of-care device used for measuring TSB with values up to $30 \mathrm{mg} / \mathrm{dL}(517 \mu \mathrm{mol} / \mathrm{L})$; it is simple to use, requiring only $25 \mu \mathrm{l}$ blood sample to load onto Bilistick Test Strips that are inserted into the battery-operated Bilistick Reader where TSB is measured by reflectance spectroscopy within $2 \mathrm{~min}$. The study demonstrates that the device has a very high "99\% accuracy and $100 \%$ sensitivity to predict laboratory TSB of $\geq 80 \mu \mathrm{mol} / \mathrm{L}$ and of $360 \mu \mathrm{mol} / \mathrm{L}$ at Bilistick TSB levels of $\geq 55 \mu \mathrm{mol} / \mathrm{L}$, and $\geq 315 \mu \mathrm{mol} / \mathrm{L}$, respectively". ${ }^{4}$ These findings confirm previous studies by Coda Zabetta et al. who demonstrated the accuracy of Bilistick for the first time in comparison to laboratory methods in 118 term infants, ${ }^{6}$ and by Greco et al. who showed the accuracy of Bilistick in comparison to TCB measurement (JM 103, Drager, Lubeck, Germany) in 126 preterm and term infants. ${ }^{7}$

The main advantages of the Bilistick System are its ease of use, lack of sample preparation or reagents, rapid accurate results, no influence from skin pigments, low cost, and portability. These characteristics make this device suitable also for outpatient follow-up of bilirubin levels during home-care visits by nurses or midwives. In addition, its potential use seems particularly interesting in low-resource primary-care facilities where, unfortunately, KSDs is still a frequent cause of mortality and neurological sequelae. Data from the Global Burden of Disease study in 2016 showed that neonatal hyperbilirubinemia accounted for 1309 deaths per 100,000 live births (seventh causes of neonatal deaths in the early-neonatal period (0-6 days)) in the world, but the burden of jaundice is highest in south Asia (seventh leading cause of neonatal mortality) and sub-Saharan Africa (eighth leading cause of neonatal mortality). ${ }^{8}$ In these areas, it appears feasible to plan an outpatient bilirubin screening program using this, or similar, low-cost portable devices and providing, as created by Boo et al., ${ }^{3}$ reference charts to guide health care providers in evaluating the need for further follow-up or hospital admission for phototherapy.

Thus, the Bilistick System represents a valuable addition to available devices for TSB measurements with similar accuracies to laboratory methods and to $T c B$, while it is portable, cheaper, and faster in comparison, but not affected by skin color and phototherapy. Furthermore, it provides immediate TSB values. The use of this device seems to be particularly suitable for postdischarge TSB measurements in jaundiced infants during homecare visits and could contribute to widespread neonatal hyperbilirubinemia screening, an essential step for an effective prevention of KSDs.

\footnotetext{
'Division of Neonatology, Careggi University Hospital of Florence, Florence, Italy and ${ }^{2}$ Department of Neurosciences, Psychology, Drug Research and Child Health, Careggi University Hospital of Florence, Florence, Italy

Correspondence: Carlo Dani (cdani@unifi.it)
}

Received: 7 February 2019 Revised: 5 March 2019 Accepted: 15 March 2019

Published online: 22 March 2019 


\section{ADDITIONAL INFORMATION}

Competing interests: The authors declare no competing interests.

Publisher's note: Springer Nature remains neutral with regard to jurisdictional claims in published maps and institutional affiliations.

\section{REFERENCES}

1. Battersby, C., Michaelides, S., Upton, M. \& Rennie, J. M. Jaundice Working Group of the Atain (Avoiding Term Admissions Into Neonatal units) programme, led by the Patient Safety team in NHS Improvement. Term admissions to neonatal units in England: a role for transitional care? A retrospective cohort study. BMJ Open 7, e016050 (2017).

2. Gale, C., Statnikov, Y., Jawad, S., Uthaya, S. N. \& Modi, N., Brain Injuries expert working group. Neonatal brain injuries in England: population-based incidence derived from routinely recorded clinical data held in the National Neonatal Research Database. Arch. Dis. Child Fetal Neonatal Ed. 103, F301-F306 (2018).

3. Le Pichon, J. B., Riordan, S. M., Watchko, J. \& Shapiro, S. M. The neurological sequelae of neonatal hyperbilirubinemia: definitions, diagnosis and treatment of the Kernicterus Spectrum Disorders (KSDs). Curr. Pediatr. Rev. 13, 199-209 (2017).

4. Borgard, J. P., Szymanowicz, A., Pellae, I., Szmidt-Adjidé, V. \& Rota, M. Determination of total bilirubin in whole blood from neonates: results from a French multicenter study. Clin. Chem. Lab Med. 44, 1103-1110 (2006).

5. Boo N. Y. et al. The point-of-care Bilistick method has very short turn-around-time and high accuracy at lower cutoff levels to predict laboratory-measured TSB. Pediatr Res. (2019). https://doi.org/10.1038/s41390-019-0304-0. [Epub ahead of print].

6. Coda Zabetta, C. D. et al. Bilistick: a low-cost point-of-care system to measure total plasma bilirubin. Neonatology 103, 177-181 (2013).

7. Greco, C. et al. Comparison between Bilistick System and transcutaneous bilirubin in assessing total bilirubin serum concentration in jaundiced newborns. J. Perinatol. 37, 1028-1031 (2017).

8. Olusanya B. O., Kaplan M., Hansen T. W. R. Neonatal hyperbilirubinaemia: a global perspective. Lancet Child Adolesc. Health 2, 610-620 (2018). 